\title{
1 pSNAP: Proteome-wide analysis of elongating nascent 2 polypeptide chains
}

4 Junki Uchiyama ', Rohini Roy ${ }^{2,3}$, Dan Ohtan Wang 2,4,5, Chiaki Yoshino ', Yuichiro

5 Mishima $^{6}$, Yasushi Ishihama ${ }^{1,7^{*}}$, Koshi Imami $1,8^{*}$

${ }^{1}$ Graduate School of Pharmaceutical Sciences, Kyoto University, Kyoto 606-8501, Japan

$8{ }^{2}$ Institute for Integrated Cell-Material Sciences (iCeMS), Kyoto University, Kyoto, 606-8501,

9 Japan

$10{ }^{3}$ Graduate School of Biostudies, Kyoto University, Kyoto, 606-8501, Japan

$11{ }^{4}$ Wuya College of Innovation, Shenyang Pharmaceutical University, 110001, China

$12{ }^{5}$ Center for Biosystems Dynamics Research, RIKEN, Kobe, Hyogo 650-0047, Japan

${ }^{6}$ Faculty of Life Sciences, Kyoto Sangyo University, Kita-ku, Kyoto 603-8555, Japan

${ }^{7}$ Laboratory of Clinical and Analytical Chemistry, National Institute of Biomedical Innovation,

15 Health and Nutrition, Ibaraki, Osaka, 567-0085, Japan

8 PRESTO, Japan Science and Technology Agency (JST), Chiyoda-ku, Tokyo, 102-0075, Japan

${ }^{*}$ Corresponding authors:

Koshi Imami

Department of Molecular and Cellular BioAnalysis, Graduate School of Pharmaceutical Sciences, Kyoto University, Kyoto 606-8501, Japan Tel.: +81-75-753-4565

Fax.: +81-75-753-4601 email: imami.koshi.3z@kyoto-u.ac.jp

28 Department of Molecular and Cellular BioAnalysis,

29 Graduate School of Pharmaceutical Sciences, Kyoto University, Kyoto 606-8501, Japan

30 Tel.: $+81-75-753-4555$

31 Fax.: +81-75-753-4601

32 email: yishiham@pharm.kyoto-u.ac.jp 


\section{Summary}

41 Cellular global translation is often measured using ribosome profiling or quantitative mass

42 spectrometry, but these methods do not provide direct information at the level of elongating

43 nascent polypeptide chains (NPCs) and associated co-translational events. Here we describe

44 pSNAP, a method for proteome-wide profiling of NPCs by affinity enrichment of puromycin- and

45 stable isotope-labeled polypeptides. pSNAP does not require ribosome purification and/or

46 chemical reaction, and captures bona fide NPCs that characteristically exhibit protein N-terminus-

47 biased positions. We applied pSNAP to evaluate the effect of silmitasertib, a potential molecular

48 therapy for cancer and COVID-19 patients, and revealed acute translational repression through

49 casein kinase II and mTOR pathways. We also characterized modifications on NPCs and

50 demonstrated that the combination of different types of modifications, such as acetylation and

51 phosphorylation in the $\mathrm{N}$-terminal region of histone $\mathrm{H} 1.5$, can modulate interactions with

52 ribosome-associated factors. Thus, pSNAP provides a framework for dissecting co-translational

53 regulations on a proteome-wide scale.

54

55

56

57

58

59

60

61

62

63

64

65

66

67

68

69

70

71

72 


\section{Introduction}

75 Co-translational regulation, such as modifications of nascent polypeptide chains (NPCs) during 76 translation, drives many aspects of cellular proteostasis, including protein folding, processing,

77 subcellular targeting, and translational control (Collart and Weiss, 2020; Schwarz and Beck, 2019).

78 Therefore, monitoring co-translational events at the NPC level is crucial for understanding cellular

79 proteome dynamics at the moment a peptide is born.

Current approaches for systematically profiling the newly synthesized proteome are mainly based on the capture of proteins metabolically labeled with stable isotope-labeled (SILAC) amino acids

83 (Doherty et al., 2009; Klann et al., 2020; Schwanhäusser et al., 2009) or bioorthogonal amino 84 acids such as azidohomoalanine (Dieterich et al., 2006; Eichelbaum et al., 2012; McShane et al., 85 2016). These methods allow us to profile mainly fully translated products (Eichelbaum et al., 2012; 86 McShane et al., 2016), but cannot enrich NPCs actively being elongated by ribosomes in action. 87 In contrast, puromycin is an aminoacylated tRNA analog that can be incorporated at the C-termini 88 of elongating NPCs (Aviner, 2020). Hence, puromycin labeling has been extensively used to monitor protein synthesis in many applications, including imaging and immunoblotting, and in various systems ranging from cell-free translation to cultured cells and whole animals (Aviner, 2020). However, the utility of puromycin or its derivatives for proteome-wide analysis of NPCs has been limited due to the need for complicated procedures, including ribosome purification by ultracentrifugation(Aviner et al., 2013) and/or chemical labeling (Forester et al., 2018; Huang et al., 2021; Tong et al., 2020; Uchiyama et al., 2020) prior to affinity purification of NPCs. background pre-existing proteins to beads or resin during affinity purification (Eichelbaum et al., 2012; Howden et al., 2013; Mellacheruvu et al., 2013).

To overcome these limitations, we have developed a method that combines quantitative proteomics and dual pulse labeling with puromycin and SILAC amino acids, termed Puromycinand SILAC labeling-based NAscent Polypeptidome profiling (pSNAP). We demonstrate the broad utility of the method by applying it to both HeLa cells and primary cells to characterize rapid translational changes as well as co-translational modifications such as protein $\mathrm{N}$-terminal acetylation. 


\section{Results and Discussion}

109 pSNAP enables global profiling of nascent polypeptide chains

110 We reasoned that NPCs incorporating puromycin could be immunoprecipitated with an anti111 puromycin antibody (Fig. 1a), thereby allowing for proteome-wide analysis of NPCs by means of 112 liquid chromatography-tandem mass spectrometry (LC/MS/MS). We first confirmed that 113 puromycin incorporation into proteins was translation-dependent (Supplementary Fig. 1a) and 114 that no marked degradation of the puromycin-labeled proteins occurred during $2 \mathrm{hr}$ treatment of 115 HeLa cells with $10 \mu \mathrm{M}$ puromycin (Supplementary Fig. 1b). We then tested a monoclonal 116 antibody against puromycin (clone 12D10) and found that puromycylated proteins could be 117 effectively immunoprecipitated when appropriate amounts of the antibody and input protein were used (Supplementary Fig. 1c). Based on these results, we used $15 \mu \mathrm{g}$ antibodies per $250 \mu \mathrm{g}$ protein input for subsequent immunoprecipitation (IP) experiments.

We next sought to profile individual NPCs with LC/MS/MS. For proof-of-concept, HeLa cells were pulse-labeled with a combination of puromycin and 'heavy (H)' amino acids (Arg'10' and Lys'8') for $2 \mathrm{hr}$ (Fig. 1b). As a control, cells were treated with only 'medium-heavy (M)' amino acids (Arg'4' and Lys'4') and puromycin was omitted. The use of ' $\mathrm{M}$ ' and 'H' labeling enabled us to distinguish bona fide NPCs (H-labeled) from non-specific proteins [light (L)- or M-labeled]. As expected, Hlabeled proteins were highly enriched in the IP sample (Fig. 1C top) while pre-existing proteins were predominantly observed in the input (Fig. 1c bottom). Overall, we observed high $\mathrm{H} / \mathrm{M}$ ratios $(\geqq 2)$ for $70 \%$ of 2,619 quantified proteins with good reproducibility (Fig. 1d and Supplementary Table 1), demonstrating that pSNAP can profile thousands of NPCs. In contrast, we observed low $\mathrm{H} / \mathrm{L}$ ratios $(<1)$ for $62 \%$ of the quantified proteins (Supplementary Fig. 1d) due to the high background resulting from non-specific binding of pre-existing proteins $(L)$ to beads. These results highlight the importance of pulse labeling with SILAC amino acids to differentiate NPCs and preexisting proteins.

Proteins captured by pSNAP exhibit a signature of elongating nascent

\section{7 polypeptides}

138 Ribosomes elongate NPCs from their amino (N-) terminal end to their carboxy (C-) terminal end 139 (Fig. 1e top). In line with this directionality, the positions of peptides with $\mathrm{H} / \mathrm{M} \geqq 2$ clearly showed 140 a bias towards the N-termini of the corresponding proteins (Fig. 1e bottom), further supporting 
141 the enrichment of elongating NPCs. Notably, such a trend was not observed for less enriched

142 peptides (i.e., $\mathrm{H} / \mathrm{M}<2$ ) or input cell lysates (Figure 1e bottom). In addition to HeLa cells, we

143 applied pSNAP to mouse primary cortical cultures, which contains a mixture of brain cell types

144 but are highly enriched for neurons (Supplementary Fig. 2a). pSNAP revealed the strong

145 enrichment of peptides near the N-termini of proteins. (Supplementary Figs. 2b, c) and achieved

146 highly sensitive detection of NPCs, including neuronal markers; NCAM1, FOXG1, and DCX,

147 supporting the validity of the results in HeLa cells (Supplementary Table 2). We also quantified

148 the differential NPC profiles between 5 and 14 days in vitro (DIV) (Supplementary Figs. 2d-f and

149 Supplementary Table 3), which overall reflected the known proteome dynamics during the

150 neuronal development in vitro(Frese et al., 2017). For example, nascent proteins related to

151 'synaptic vesicle cycle' were overrepresented in DIV14 (Supplementary Fig. 2f), consistent with

152 the occurrence of synapse formation and maintenance after development. Collectively, these

153 results indicate that pSNAP captures genuine NPCs and enables quantitative nascent proteome

154 analysis.

\section{Quantifying translational responses induced by a kinase inhibition}

158 The ability to capture and quantify NPCs proteome-wide with high accuracy enables quantitative 159 measurements of acute translational changes that allow cells to respond to specific stimuli. To 160 illustrate this, we next applied pSNAP to characterize the global impact of a kinase inhibitor on 161 cellular translation. We focused on casein kinase 2 (CK2) as it is ubiquitously expressed in all 162 cells, and has been implicated in translational control through phosphorylation of specific 163 eukaryotic initiation factors (elFs) (Gandin et al., 2016; Lamper et al., 2020). However, the protein 164 targets of translational regulation by CK2 remain unknown. Therefore, HeLa cells were pre165 incubated with either DMSO or a specific CK2 inhibitor silmitasertib (also known as CX4945) 166 (Siddiqui-Jain et al., 2010) for $10 \mathrm{~min}$ (Fig. 2a), and then pulse-labeled with puromycin and SILAC 167 amino acids for $2 \mathrm{hr}$ in the presence of DMSO or silmitasertib, and processed as illustrated in Fig. 168 1b. H/M ratios in MS spectra represent the difference in production of NPCs between the two 169 conditions (silmitasertib and DMSO treatments). We first confirmed that NPCs could be enriched: $170 \mathrm{H}$ - and $\mathrm{M}$-labeled peptides both exhibited the trend of protein $\mathrm{N}$-terminus-biased positions 171 (Supplementary Fig. 3a).

173 To understand the gene expression networks at the NPC level, we first asked whether in vitro 174 CK2 substrates (Sugiyama et al., 2019) might be regulated translationally but found no evidence 
175 for that (Fig. 2b). On the one hand, we found that CK2 inhibition led to marked repression of

176 NPCs with a $5^{\prime}$ terminal oligopyrimidine (TOP) motif in their mRNAs ( $\left.p=5.7 e-16\right)$, encoding for

177 components of the translational machinery (Fig. 2b and Supplementary Fig. 3b). TOP mRNAs

178 are well-known targets that are subject to selective translation through mTORC1 (Hsieh et al.,

179 2012; Thoreen et al., 2012). Hence, CK2 may regulate the translation of TOP mRNAs in concert

180 with mTORC1, in line with a previous report that CK2 enhances mTORC1 activity (Gandin et al.,

181 2016). In addition, pSNAP revealed that the translation of specific subsets of proteins (e.g.,

182 RCOR1, RAE1, and RNPS1) was down-regulated (Supplementary Table 4). Thus, our analysis

183 uncovered acute translational responses to silmitasertib via the CK2 and mTOR pathways, which

184 may contribute to further understanding of the mechanism of action of silmitasertib, a promising

185 molecular therapy for several types of cancers (phase II) (Siddiqui-Jain et al., 2010) as well as

186 SARS-CoV-2 infection (Bouhaddou et al., 2020).

\section{Profiling modifications on nascent proteins}

The present method not only enables the global profiling of NPCs, but also highlights the cotranslationally regulated modifications of NPCs. Conventional proteomic approaches cannot resolve post- and co-translational modifications, and so the distribution of the two types of modifications within a protein goes undetected; yet the timing of modifications can be important for protein processing (Aksnes et al., 2019; Varland et al., 2015) such as folding (Keshwani et al., 2012; Kii et al., 2016). By detecting NPCs with their modification states, pSNAP can be particularly useful in revealing co-translational modifications. In the silmitasertib treatment experiment (Fig. 2a), we found that CK2 inhibition led to decreased phosphorylation levels on nascent forms of known CK2 substrates, such as G3BP1 pS149 (Reineke et al., 2017), HSP90AA1 pS231, and HSP90AB1 pS226, pS255 (Mollapour and Neckers, 2012), while no marked change was observed at the NPC level (Fig. 2c and Supplementary Table 4). Hence, CK2 may act in close proximity to the ribosome to co-translationally phosphorylate nascent proteins, possibly regulating protein stability through phosphorylation of newly made proteins, as observed for XRCC1 (Parsons et al., 2010) and CFTR (Pankow et al., 2019).

Protein N-terminal (Nt) acetylation is one well-studied 'co-translational' modification (Aksnes et al., 2019; Yeom et al., 2017); however, recent studies have revealed 'post-translational' Ntacetylation on many transmembrane proteins and actin (Yeom et al., 2017). While earlier N- 
Lai et al., 2015; Yeom et al., 2017), it remains unclear whether these sites are co- or posttranslationally modified. We thus sought to apply pSNAP to pinpoint co-translational Ntacetylation sites with high accuracy. For this purpose, HeLa cells were treated with either $\mathrm{CHX}$ or DMSO for $2 \mathrm{hr}$ in the presence of puromycin and corresponding SILAC amino acid pairs. By combining PSNAP with low pH strong cation exchange (SCX) chromatography (Helbig et al.,

214 2010), we enriched Nt-acetylated peptides that were eluted in the flow-through fraction and early

215 in SCX fractionation due to the loss of positive charge at their $\mathrm{N}$-terminal ends (Fig. 3a). We

216 confirmed that the NPCs could be enriched (Supplementary Figs. 4a, b and Supplementary

217 Table 5) and identified 298 unique protein Nt-acetylated sites that exhibited H/M (DMSO/CHX)

$218 \geqq 2$ in at least one of the three replicates. Of note, beta-actin's Nt-acetylation showed $H / M<1$ in

219 all replicates, indicating that it occurs post-translationally, in agreement with a previous report 220 (Drazic et al., 2018). To better understand acceptor sites for co-translational Nt-acetylation, we 221 focused on amino acids at the second residue [next to the initiator methionine (iMet)] (Fig. 3b). In 222 accordance with the substrate specificity of major N-terminal acetyltransferases (Aksnes et al., 223 2019; Yeom et al., 2017), we observed a high prevalence of alanine and serine for Nt-acetylated 224 NPCs whose iMet was cleaved ( $p<0.01$, v.s. amino acid frequency at the second residue of the 225 human proteome), while the acidic amino acids (aspartic acid and glutamic acid) and phenylalanine were overrepresented in the iMet-retained and Nt-acetylated NPCs $(p<0.01)$ (Fig. 3b). Hence, these specific amino acids at the $\mathrm{N}$-termini might be key determinants in regulating a protein's life and function at birth(Timms et al., 2019).

\section{Protein $\mathrm{N}$-terminal modifications on a nascent protein can modulate binding} partners

Protein $\mathrm{N}$-termini are hotspots for modifications during translation and thus can regulate cotranslational events such as folding and degradation through interactions with proteins (Collart and Weiss, 2020). We next sought to discover cross-talk between protein Nt-acetylation and other modifications on NPCs as the combination of different types of modifications confers additional specificity and combinatorial logic to protein interactions. We searched our dataset focusing on phosphorylation, which can function as a versatile switch to modulate protein interactions. We identified 134 phosphorylated peptides in the Nt-acetylation-enriched samples. Among them, we focused on phosphorylation at Ser19 of histone H1.5 (H1.5) co-occurring with protein Nt-

242 indicated that the Nt-acetylated form is a major nascent proteoform of H1.5 (Fig. 3d). Such highly 
stoichiometric patterns of nascent Nt-acetylation were also seen for the 13 sites whose Ntacetylated and its counterpart (unmodified) peptides were both quantified (Fig. 3e), in marked contrast to the very low (median $0.02 \%$ ) stoichiometry of lysine acetylation in HeLa cells (Hansen et al., 2019).

To understand the role of the nascent modifications and their impacts on protein interactions, we performed peptide-based pulldown experiments on HeLa cell lysate using three distinct peptide probes that mimic 1) Nt-unmodified, 2) Nt-acetylated, or 3) Nt-acetylated and phosphorylated forms of H1.5 (Fig. 3f). The peptide-based screen revealed proteins that differentially interacted with the specific peptide probes (Fig. $\mathbf{3 g}$ and Supplementary Table 6). One prominent example is a ubiquitin E3 ligase complex (KLHL13, KLHL22, CUL3, BIRC6) that showed a robust interaction with the unmodified peptides (Fig. $\mathbf{3 g}$ left). Thus, Nt-unmodified H1.5 is likely to be degraded through the ubiquitin-proteasome system, which may explain why the Nt-unmodified H1.5 was markedly less abundant than the acetylated forms in HeLa cells (Fig. 3d). Accordingly, protein Nt-acetylation of $\mathrm{H} 1.3$ is protective against protein degradation, in line with the idea that Nt-acetylated mitochondrial proteins bearing inhibitor of apoptosis binding (IAP) motifs are shielded from the IAP family of E3 ubiquitin ligases(Mueller et al., 2021). Interestingly, the Ntacetylated and phosphorylated version of the peptide preferentially bound a co-translational quality control factor PELOTA while disfavoring the interaction with a molecular chaperone HSPA6 (Fig. 3g right). PELOTA was shown to promote the dissociation of stalled ribosomes and the release of intact peptidyl-tRNA for ribosome recycling (Pisareva et al., 2011); Thus, the nascent $\mathrm{H} 1.5$ phosphorylation in the $\mathrm{N}$-terminal region may represent an additional 'modification code' to recruit PELOTA and to repel HSPA6 as a surveillance mechanism for aberrant nascent H1.5. In summary, pSNAP enables us to uncover modifications on NPCs that may represent a new layer of translational control, i.e., one shaped by nascent protein modifications.

\section{Limitations of the method}

271 While puromycin or its analogue has been used to analyze protein synthesis in many cell lines 272 and model systems (Aviner, 2020), it may also cause a secondary effect on cellular translation

273 (Marciano et al., 2018). In this study, we therefore used a relatively low concentration of puromycin 274 that did not affect cell viability or degradation of puromycylated proteins at least in HeLa cells, but 275 further investigations would be required to characterize the mode of action of puromycin in detail. 276 We demonstrated that pSNAP can be readily applicable to a cell line and primary-cultured cells, 
277 though further development will be needed for its application to in vivo systems. For example, the

278 direct enrichment and proteomic analysis of puromycylated peptides digested from NPCs

279 provides a signature of genuine NPCs, and would not require pulse SILAC labeling, which is not

280 generally feasible in animals.

\section{Conclusions}

284 The pSNAP approach presented here offers multiple advantages over currently available 285 methods for capturing NPCs with co-translational modifications, a hidden layer in understanding 286 translational regulations in cell biology, but are inaccessible by conventional ribosome profiling or 287 proteomic approaches. The advantage of pSNAP lies in the use of dual pulse labeling. The 288 incorporation of puromycin facilitates the enrichment of NPCs from a complex background, and 289 in turn, the use of pulsed SILAC enables both protein quantification and the ability to discriminate 290 nascent from pre-existing proteins. Moreover, the experimental workflow is simple in contrast to 291 existing methods that involve ribosome purification using ultracentrifugation(Aviner et al., 2013) 292 and/or chemical labeling steps (Forester et al., 2018; Huang et al., 2021; Tong et al., 2020; 293 Uchiyama et al., 2020). In addition, the method does not require special puromycin derivatives 294 such as biotin-puromycin (Aviner et al., 2013)or clickable puromycin(Forester et al., 2018; Huang 295 et al., 2021; Tong et al., 2020; Uchiyama et al., 2020). Our results show that pSNAP can quantify 296 changes in NPC levels in response to environmental cues, and is useful for characterizing cotranslational modifications. It might also be employed in combination with biochemical enrichment techniques for specific modified forms of peptides/proteins. In addition, this method could be applied to identify the NPC interactome during translation since some proteins appear to form homo- (Bertolini et al., 2021) or hetero- (Kamenova et al., 2019) complexes in a co-translational manner. 


\section{Figure legends}

312 Fig. 1 Profiling nascent polypeptide chains by pSNAP.

313 a, Principle of the enrichment of NPCs with the PSNAP. $\mathbf{b}$, Schematic representation of pSNAP 314 workflow. HeLa cells are pulse labeled for $2 \mathrm{hr}$ with a combination of $10 \mu \mathrm{M}$ puromycin and heavy 315 amino acids or with only medium-heavy amino acids. After IP of NPCs with anti-puromycin 316 antibodies, NPCs are eluted with $0.15 \%$ TFA and digested into tryptic peptides. The resulting 317 peptide sample is analyzed by LC/MS/MS. Heavy-to-medium $(H / M)$ ratios represent the degree 318 of enrichment of NPCs. c, Exemplary MS spectra for AAALEFLNR (STIP1) obtained with (top) 319 and without (bottom) enrichment. The isotope clusters of the $L, M$, and $H$ peaks correspond to 320 non-specific proteins from the pre-existing proteome pool and from the 'medium'-labeled proteins and 'heavy'-labeled NPCs, respectively. d, Multi-scatter plots of $\log _{2} \mathrm{H} / \mathrm{M}$ ratios from three independent experiments. e, pSNAP can enrich NPCs. (top) The ribosome elongates an NPC from its $\mathrm{N}$-terminal end to the $\mathrm{C}$-terminal end. Thus, positions of NPC-derived tryptic peptides are biased towards the $\mathrm{N}$-termini of proteins. (bottom) Relative starting positions of identified peptides within proteins. The bars represent averaged values from three independent experiments.

\section{Fig. 2 Quantitative analysis of translational responses and co-translational} phosphorylation.

a, Experimental design for global analysis of translational changes induced by a CK2 inhibitor silmitasertib $(10 \mu \mathrm{M})$. b, A histogram of $\log _{2}$ fold changes [H/M (silmitasertib/DMSO) ratios] of protein synthesis induced by silmitasertib. Averaged $\mathrm{H} / \mathrm{M}$ ratios based on proteins quantified in at least two of the three replicates are shown. Results from individual replicates are shown in Supplementary Fig. 3b. A subset of proteins whose mRNAs contain a TOP motif is shown in dark blue. CK2 substrates determined by an in vitro kinase reaction(Sugiyama et al., 2019) are shown in orange. All quantified proteins are shown in black. The dashed lines indicate median values of the three groups (all, TOP motif, and CK2 substrates) The $p$-value was computed using the two-sided Wilcoxon rank-sum test. c, Change of NPC and phosphorylation levels due to silmitasertib treatment versus DMSO treatment. The indicated phosphorylation sites of G3BP1, HSP90AA1 and HSP90AB1 are known CK2 substrates. Levels of the NPCs were quantified from NPC-derived unphosphorylated peptides. The bars represent averaged values from two or three 341 replicates. 
344 a, Enrichment of Nt-acetylated peptides with SCX-based fractionation. The percentage of the 345 number of protein Nt-acetylated peptides in all peptides identified in individual fractions is shown.

346 The bars represent averaged values from three replicates. $\mathbf{b}$, The amino acid frequency at the 347 second residue next to iMet of acetylated protein $\mathrm{N}$-termini based on the absence (pink) or 348 presence (light green) of iMet. Only nascent proteins that showed $\mathrm{H} / \mathrm{M}>2$ in at least one of the 349 three replicates were considered. For comparison, the human proteome from the SwissProt 350 protein database is shown. The p-value was computed using Fisher's exact test. c, 351 Representative MS/MS spectra of Ac-SETAPAETATPAPVEK (left) and Ac352 SETAPAETATPAPVEKPSPAK (right) from histone H1.5. The insets show representative MS spectra of corresponding peptides and demonstrate that the protein Nt-acetylation and adjacent phosphorylation occurred on nascent H1.5. d, The comparison of H-labeled peptide intensities from Nt-acetylated (red), Nt-acetylated and phosphorylated (orange), and Nt-unmodified (grey) forms. e, Stoichiometry (\%) of protein Nt-acetylation estimated based on intensities of H-labeled peptides from Nt-acetylated and counterpart (unmodified) forms. The bars represent averaged values from two or three replicates. f, Experimental design for peptide-pulldown assays using three different peptide probes. Nt-unmodified, Nt-acetylated, or Nt-acetylated and phosphorylated biotinylated peptides corresponding to amino acid residues from 2 to 22 (SETAPAETATPAPVEKSPAKK) of $\mathrm{H} 1.5$ were conjugated to streptavidin agarose resins. Beads were incubated with HeLa cell lysate, and eluted for LC/MS/MS analysis. g, Volcano plots from the pulldown assays of Nt-free vs Nt-acetylated peptides (left) and Nt-acetylated vs Nt-acetylated and phosphorylated peptides (right) are shown.

Supplementary Fig. 1 Assessment of puromycin labeling and immunoprecipitation of puromycylated proteins.

a, Puromycin labeling of proteins is translation-dependent. Western blots (left) and Ponceau staining (right) of whole-cell lysates of HeLa cells treated with $10 \mu \mathrm{M}$ puromycin for $2 \mathrm{hr}$, or $10 \mu \mathrm{M}$ puromycin and $\mathrm{CHX}$ for $2 \mathrm{hr}$, or neither. $\mathbf{b}$, Abundance of puromycylated proteins was not altered in the presence of a proteasome inhibitor (10 $\mu \mathrm{M}$ MG132 or $1 \mu \mathrm{M}$ bortezomib) over $2 \mathrm{hr}$ treatment.

373 Western blotting was performed with anti-puromycin antibody (left), anti-ubiquitin antibody 374 (middle), and Ponceau staining (right). This result indicates that puromycylated NPCs were not 375 significantly degraded via the ubiquitin-proteasome system in this time frame, while levels of 376 ubiquitinated total cellular proteins were increased in the presence of proteasome inhibitors. c, 377 Puromycylated proteins can be immunoprecipitated. Immunoprecipitation (IP) was performed for 
different protein inputs $(50,100,250$, and $500 \mu \mathrm{g})$ with a fixed amount of $15 \mu \mathrm{g}$ anti-puromycin antibody. The proteins eluted with $0.15 \%$ TFA and the remaining supernatant in a post-IP sample were analyzed by means of immunoblotting with the anti-puromycin antibody. 50-250 $\mu \mathrm{g}$ protein inputs were found to be suitable for immunoprecipitation with $15 \mu \mathrm{g}$ anti-puromycin antibodies. $\mathbf{d}$, Multi-scatter plots of $\log _{2} \mathrm{H} / \mathrm{L}$ ratios from three independent experiments of puromycin $(\mathrm{H})$ and no-puromycin (L) treatments.

\section{Supplementary Fig. 2 Application of pSNAP to mouse primary cortex culture.}

a, Schematic representation of the pSNAP workflow. Mouse primary cortical neurons are pulse labeled for $2 \mathrm{hr}$ with a combination of $10 \mu \mathrm{M}$ puromycin and heavy amino acids or only mediumheavy amino acids. After IP of NPCs with anti-puromycin antibodies, NPCs are eluted with $0.15 \%$ TFA and digested into tryptic peptides. The resulting peptide sample is analyzed by LC/MS/MS. b, Multi-scatter plots of $\log _{2} \mathrm{H} / \mathrm{M}$ ratios from three independent experiments using mouse primary neurons. c, pSNAP can enrich NPCs from primary neurons. Relative starting positions of identified peptides within proteins for enriched (left) or input (right) samples. The bars represent averaged values from three independent experiments. $\mathbf{d}$, Experimental design for the differential nascent proteome profiling of the mouse primary cultures between DIV 5 and 14. e, A volcano plot showing differential NPC levels between DIV 5 and 14. $\log _{2}$ FC (H/M: DIV14/DIV5) and pvalues are computed from three biological replicates (see method). f, Gene ontology enrichment analyses for the significantly regulated NPCs $\left(\log _{2} \mathrm{FC}>0.5\right.$ and $\left.p<0.05\right)$.

Supplementary Fig. 3 Quantitative analysis of translational changes associated with CX4945 treatment.

a, pSNAP can enrich NPCs from HeLa cells treated with DMSO or CX-4945. Relative starting positions of identified peptides (only M- or H-labeled) within proteins for NPC-enriched samples. The bars represent averaged values from three independent experiments. $\mathbf{b}$, Histogram of $\log _{2}$ FCs [H/M (silmitasertib/DMSO) ratios] of protein synthesis induced by silmitasertib. Results from individual replicates are shown. A subset of proteins whose mRNAs contain a TOP motif is shown in light blue. All quantified proteins are shown in pink. The p values were computed using twosided Wilcoxon rank-sum test.

410 a, Multi-scatter plots of $\log _{2} \mathrm{H} / \mathrm{M}$ ratios from three independent experiments using HeLa cells 411 treated with DMSO or CHX. b, pSNAP can enrich NPCs. Relative starting positions of identified 
412 peptides within proteins for enriched (left) or input (right) samples. The bars represent averaged

413 values from three independent experiments.

\section{Acknowledgements}

416 We are very grateful to Erik McShane (Harvard Medical School), Matthew L Kraushar (Charité-

417 Universitätsmedizin Berlin), and Tatsuya Niwa (Tokyo Institute of Technology) for critical reading

418 of the manuscript. We thank the members of the Department of Molecular \& Cellular BioAnalysis

419 and the Department of Proteomics, Drug Discovery for fruitful discussion. KI thanks the Samuro

420 Kakiuchi Memorial Research Award for Young Scientists for supporting this study. This work was

421 supported by JSPS Grant-in-Aid for Scientific Research (Grant Numbers JP18K14674,

$422 \mathrm{JP2OH} 03241$, JP2OH04844 to $\mathrm{KI}$ and $17 \mathrm{H} 05667$ to $\mathrm{YI}$ ), JST PRESTO (JPMJPR18H2), the

423 Takeda Science Foundation to KI, AMED (18dm0307023h) to DOW, JST Strategic Basic

424 Research Program CREST (18070870), and AMED Advanced Research and Development

425 Programs for Medical Innovation CREST (18068699) to YI.

\section{Author contributions}

428 Conceptualization \& Methodology, J.U., Y.I., and K.I.; Investigation, J.U., R.R., Y.M., D.O.W., 429 Y.C, Y. I., and K.I; Resources, D.O.W., Y.M., Y.I., and K.I.; Writing - Original Draft, K.I; Writing -

430 Review \& Editing, J.O., R.R., Y.M, D.O.W., Y.I., Supervision, Y.I. and K.I..

433 The authors declare no competing financial interest.

\section{Supplementary information}

\section{Supplementary Figs. 1-4}

437 Supplementary Table 1 (related to Fig. 1): LC/MS/MS proteomic analysis of NPCs from HeLa

438 cells treated with $(\mathrm{H})$ or without $(\mathrm{M})$ puromycin.

439 Supplementary Table 2 (related to Supplementary Fig. 2b): LC/MS/MS proteomic analysis of

$440 \quad$ NPCs from mouse primary cells treated with $(\mathrm{H})$ or without $(\mathrm{M})$ puromycin.

441 Supplementary Table 3 (related to Supplementary Fig. 2b): LC/MS/MS differential proteomic

442 analysis of NPCs from mouse primary cells between DIV5 (M) and DIV14 (H). Statistical

443 comparisons were made using two-sided Student's t-test. 
444 Supplementary Table 4 (related to Fig. 2b): LC/MS/MS differential proteomic analysis of

445 NPCs from HeLa cells treated with DMSO $(\mathrm{M})$ or silmitasertib $(\mathrm{H})$. Statistical comparisons were

446 made using two-sided Wilcoxon rank-sum test.

447 Supplementary Table 5 (related to Fig. 3b): LC/MS/MS differential proteomic analysis of

448 NPCs from HeLa cells treated with $\mathrm{CHX}(\mathrm{M})$ or DMSO $(\mathrm{H})$. Unique protein Nt-acetylated sites

449 that exhibit $\mathrm{H} / \mathrm{M} \geqq 2$ are also shown.

450 Supplementary Table 6 (related to Fig. $3 \mathrm{~g}$ ): LC/MS/MS differential proteomic analysis of

451 peptide-based pulldown experiments on HeLa cells. Statistical comparisons were made using

452 two-sided Student's t-test.

References

Aksnes, H., Ree, R., and Arnesen, T. (2019). Co-translational, Post-translational, and Noncatalytic Roles of N-Terminal Acetyltransferases. Mol. Cell 73, 1097-1114.

Aviner, R. (2020). The science of puromycin: From studies of ribosome function to applications in biotechnology. Comput. Struct. Biotechnol. J. 18, 1074-1083.

Aviner, R., Geiger, T., and Elroy-Stein, O. (2013). Novel proteomic approach (PUNCH-P) reveals cell cycle-specific fluctuations in mRNA translation. Genes Dev. 27, 1834-1844.

Bertolini, M., Fenzl, K., Kats, I., Wruck, F., Tippmann, F., Schmitt, J., Auburger, J.J., Tans, S., Bukau, B., and Kramer, G. (2021). Interactions between nascent proteins translated by adjacent ribosomes drive homomer assembly. Science 371, 57-64.

Bouhaddou, M., Memon, D., Meyer, B., White, K.M., Rezelj, V.V., Correa Marrero, M., Polacco, B.J., Melnyk, J.E., Ulferts, S., Kaake, R.M., et al. (2020). The Global Phosphorylation Landscape of SARS-CoV-2 Infection. Cell 182, 685-712.e19.

Choudhary, C., Weinert, B.T., Nishida, Y., Verdin, E., and Mann, M. (2014). The growing landscape of lysine acetylation links metabolism and cell signalling. Nat. Rev. Mol. Cell Biol. 15, 536-550.

473 Collart, M.A., and Weiss, B. (2020). Ribosome pausing, a dangerous necessity for co474 translational events. Nucleic Acids Res. 48, 1043-1055.

478 Doherty, M.K., Hammond, D.E., Clague, M.J., Gaskell, S.J., and Beynon, R.J. (2009). Turnover 
481

482

483

484

485

486

487

488

489

490

491

492

493

494

495

496

497

498

499

500

501

502

503

504

505

506

507

508

509

510

511

512

513

514

515

516

517

518

519

520

Drazic, A., Aksnes, H., Marie, M., Boczkowska, M., Varland, S., Timmerman, E., Foyn, H., Glomnes, N., Rebowski, G., Impens, F., et al. (2018). NAA80 is actin's N-terminal acetyltransferase and regulates cytoskeleton assembly and cell motility. Proc. Natl. Acad. Sci. U. S. A. $115,4399-4404$.

Eichelbaum, K., Winter, M., Berriel Diaz, M., Herzig, S., and Krijgsveld, J. (2012). Selective enrichment of newly synthesized proteins for quantitative secretome analysis. Nat. Biotechnol. 30, 984-990.

Forester, C.M., Zhao, Q., Phillips, N.J., Urisman, A., Chalkley, R.J., Oses-Prieto, J.A., Zhang, L., Ruggero, D., and Burlingame, A.L. (2018). Revealing nascent proteomics in signaling pathways and cell differentiation. Proc. Natl. Acad. Sci. U. S. A. 115, 2353-2358.

Frese, C.K., Mikhaylova, M., Stucchi, R., Gautier, V., Liu, Q., Mohammed, S., Heck, A.J.R., Altelaar, A.F.M., and Hoogenraad, C.C. (2017). Quantitative Map of Proteome Dynamics during Neuronal Differentiation. Cell Rep. 18, 1527-1542.

Gandin, V., Masvidal, L., Cargnello, M., Gyenis, L., McLaughlan, S., Cai, Y., Tenkerian, C., Morita, M., Balanathan, P., Jean-Jean, O., et al. (2016). mTORC1 and CK2 coordinate ternary and elF4F complex assembly. Nat. Commun. 7, 11127.

Hansen, B.K., Gupta, R., Baldus, L., Lyon, D., Narita, T., Lammers, M., Choudhary, C., and Weinert, B.T. (2019). Analysis of human acetylation stoichiometry defines mechanistic constraints on protein regulation. Nat. Commun. 10, 1055.

Helbig, A.O., Gauci, S., Raijmakers, R., van Breukelen, B., Slijper, M., Mohammed, S., and Heck, A.J.R. (2010). Profiling of $\mathrm{N}$-acetylated protein termini provides in-depth insights into the $\mathrm{N}$-terminal nature of the proteome. Mol. Cell. Proteomics 9, 928-939.

Howden, A.J.M., Geoghegan, V., Katsch, K., Efstathiou, G., Bhushan, B., Boutureira, O., Thomas, B., Trudgian, D.C., Kessler, B.M., Dieterich, D.C., et al. (2013). QuaNCAT: quantitating proteome dynamics in primary cells. Nat. Methods $10,343-346$.

Hsieh, A.C., Liu, Y., Edlind, M.P., Ingolia, N.T., Janes, M.R., Sher, A., Shi, E.Y., Stumpf, C.R., Christensen, C., Bonham, M.J., et al. (2012). The translational landscape of mTOR signalling steers cancer initiation and metastasis. Nature 485, 55-61.

Huang, Y., Zhang, Q., Yang, L., Lin, L., Xie, J., Yao, J., Zhou, X., Zhang, L., Shen, H., and Yang, P. (2021). Puromycin-Modified Silica Microsphere-Based Nascent Proteomics Method for Rapid and Deep Nascent Proteome Profile. Anal. Chem. 93, 6403-6413.

Kamenova, I., Mukherjee, P., Conic, S., Mueller, F., El-Saafin, F., Bardot, P., Garnier, J.-M., Dembele, D., Capponi, S., Timmers, H.T.M., et al. (2019). Co-translational assembly of mammalian nuclear multisubunit complexes. Nat. Commun. 10, 1740.

Keshwani, M.M., Klammt, C., von Daake, S., Ma, Y., Kornev, A.P., Choe, S., Insel, P.A., and Taylor, S.S. (2012). Cotranslational cis-phosphorylation of the $\mathrm{COOH}$-terminal tail is a key priming step in the maturation of cAMP-dependent protein kinase. Proc. Natl. Acad. Sci. U. S. A. 109, E1221-E1229.

Kii, I., Sumida, Y., Goto, T., Sonamoto, R., Okuno, Y., Yoshida, S., Kato-Sumida, T., Koike, Y., Abe, M., Nonaka, Y., et al. (2016). Selective inhibition of the kinase DYRK1A by targeting its 
folding process. Nat. Commun. 7, 11391.

522

523

524

525

526

527

528

529

530

531

532

533

534

535

536

537

538

539

540

541

542

543

544

545

546

547

548

549

550

551

552

553

554

555

556

557

558

Klann, K., Tascher, G., and Münch, C. (2020). Functional Translatome Proteomics Reveal Converging and Dose-Dependent Regulation by mTORC1 and elF2a. Mol. Cell 77, 913925.e4.

Lai, Z.W., Petrera, A., and Schilling, O. (2015). Protein amino-terminal modifications and proteomic approaches for N-terminal profiling. Curr. Opin. Chem. Biol. 24, 71-79.

Lamper, A.M., Fleming, R.H., Ladd, K.M., and Lee, A.S.Y. (2020). A phosphorylation-regulated elF3d translation switch mediates cellular adaptation to metabolic stress. Science 370, 853856.

Marciano, R., Leprivier, G., and Rotblat, B. (2018). Puromycin labeling does not allow protein synthesis to be measured in energy-starved cells. Cell Death Dis. 9, 1-3.

McShane, E., Sin, C., Zauber, H., Wells, J.N., Donnelly, N., Wang, X., Hou, J., Chen, W., Storchova, Z., Marsh, J.A., et al. (2016). Kinetic Analysis of Protein Stability Reveals AgeDependent Degradation. Cell 167, 803-815.e21.

Mellacheruvu, D., Wright, Z., Couzens, A.L., Lambert, J.-P., St-Denis, N.A., Li, T., Miteva, Y.V., Hauri, S., Sardiu, M.E., Low, T.Y., et al. (2013). The CRAPome: a contaminant repository for affinity purification-mass spectrometry data. Nat. Methods 10, 730-736.

Mollapour, M., and Neckers, L. (2012). Post-translational modifications of Hsp90 and their contributions to chaperone regulation. Biochim. Biophys. Acta 1823, 648-655.

Mueller, F., Friese, A., Pathe, C., da Silva, R.C., Rodriguez, K.B., Musacchio, A., and Bange, T. (2021). Overlap of NatA and IAP substrates implicates $\mathrm{N}$-terminal acetylation in protein stabilization. Sci Adv 7.

Pankow, S., Bamberger, C., and Yates, J.R., 3rd (2019). A posttranslational modification code for CFTR maturation is altered in cystic fibrosis. Sci. Signal. 12.

Parsons, J.L., Dianova, I.I., Finch, D., Tait, P.S., Ström, C.E., Helleday, T., and Dianov, G.L. (2010). XRCC1 phosphorylation by CK2 is required for its stability and efficient DNA repair. DNA Repair 9, 835-841.

Pisareva, V.P., Skabkin, M.A., Hellen, C.U.T., Pestova, T.V., and Pisarev, A.V. (2011). Dissociation by Pelota, Hbs 1 and $\mathrm{ABCE} 1$ of mammalian vacant $80 \mathrm{~S}$ ribosomes and stalled elongation complexes. EMBO J. 30, 1804-1817.

Reineke, L.C., Tsai, W.-C., Jain, A., Kaelber, J.T., Jung, S.Y., and Lloyd, R.E. (2017). Casein Kinase 2 Is Linked to Stress Granule Dynamics through Phosphorylation of the Stress Granule Nucleating Protein G3BP1. Mol. Cell. Biol. 37.

Schwanhäusser, B., Gossen, M., Dittmar, G., and Selbach, M. (2009). Global analysis of cellular protein translation by pulsed SILAC. Proteomics 9, 205-209.

Schwarz, A., and Beck, M. (2019). The Benefits of Cotranslational Assembly: A Structural Perspective. Trends Cell Biol. 29, 791-803.

Siddiqui-Jain, A., Drygin, D., Streiner, N., Chua, P., Pierre, F., O’Brien, S.E., Bliesath, J., Omori, 
559 M., Huser, N., Ho, C., et al. (2010). CX-4945, an orally bioavailable selective inhibitor of protein kinase CK2, inhibits prosurvival and angiogenic signaling and exhibits antitumor efficacy.

562 Sugiyama, N., Imamura, H., and Ishihama, Y. (2019). Large-scale Discovery of Substrates of 563 the Human Kinome. Sci. Rep. 9, 10503.

564 Thoreen, C.C., Chantranupong, L., Keys, H.R., Wang, T., Gray, N.S., and Sabatini, D.M. (2012). 565 A unifying model for mTORC1-mediated regulation of mRNA translation. Nature 485, 109-113.

566 Timms, R.T., Zhang, Z., Rhee, D.Y., Harper, J.W., Koren, I., and Elledge, S.J. (2019). A glycine567 specific $\mathrm{N}$-degron pathway mediates the quality control of protein -myristoylation. Science 365.

568 Tong, M., Suttapitugsakul, S., and Wu, R. (2020). Effective Method for Accurate and Sensitive 569 Quantitation of Rapid Changes of Newly Synthesized Proteins. Anal. Chem. 92, 10048-10057.

570 Uchiyama, J., Ishihama, Y., and Imami, K. (2020). Quantitative nascent proteome profiling by 571 dual pulse labeling with O-propargyl-puromycin and stable isotope labeled amino acids. J.

572 Biochem.

573 Varland, S., Osberg, C., and Arnesen, T. (2015). N-terminal modifications of cellular proteins:

574 The enzymes involved, their substrate specificities and biological effects. Proteomics 15, 23855752401.

576 Yeom, J., Ju, S., Choi, Y., Paek, E., and Lee, C. (2017). Comprehensive analysis of human 577 protein N-termini enables assessment of various protein forms. Sci. Rep. 7, 6599. 
Figure 1

a

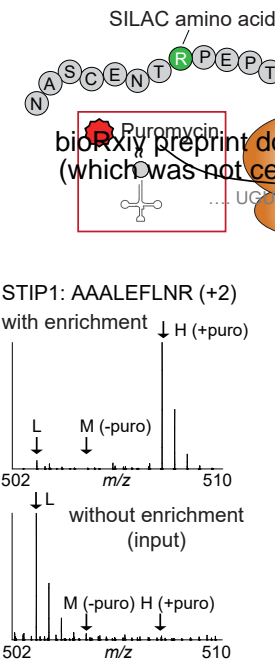

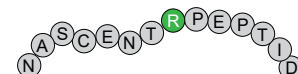
(A) Immunoprecipitation b

HeLa or mouse primary cortex cultures $(n=3)$
$0.15 \%$ TFA elution Protein digestion

\section{Light}

://doi.org/10.1101/2021. Fon 22.461445; this version posted September 23, 2021. The copyright holder for this prepritht

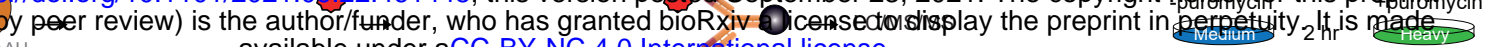
available under aCC-BY-NC 4.0 Internátional license.

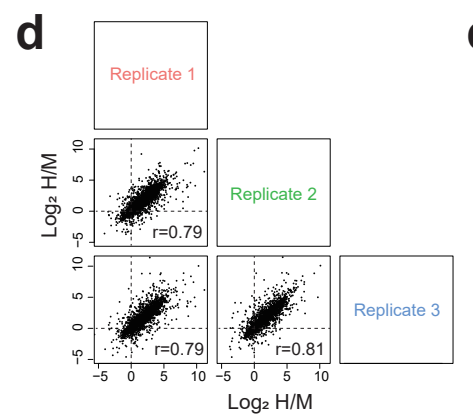

e
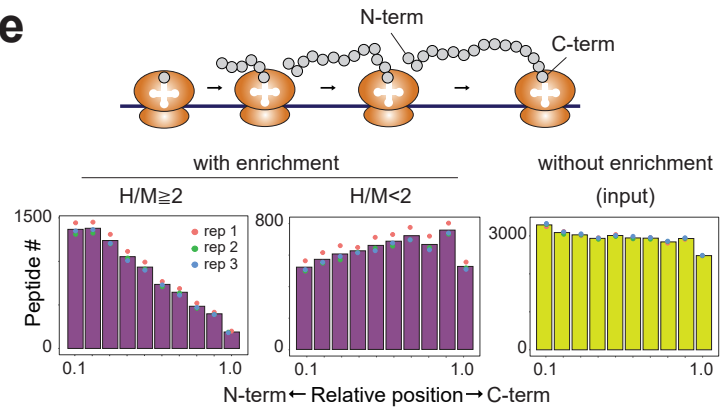
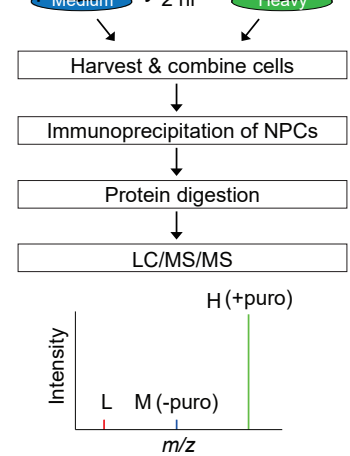

Fig. 1 Profiling elongating nascent polypeptide chains by $\mathrm{pSNAP}$.

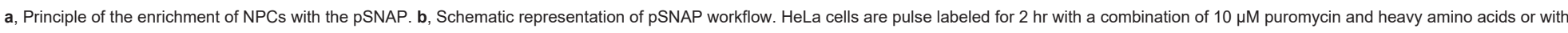

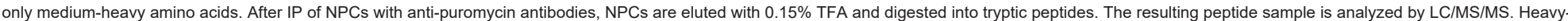

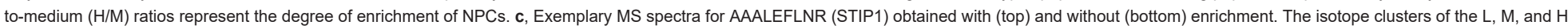

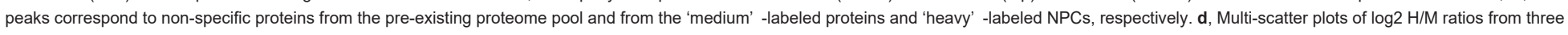

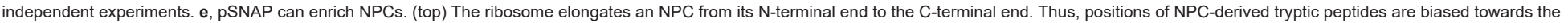
$\mathrm{N}$-termini of proteins. (bottom) Relative starting positions of identified peptides within proteins. The bars represent averaged values from three independent experiments.

\section{Figure 2}

a

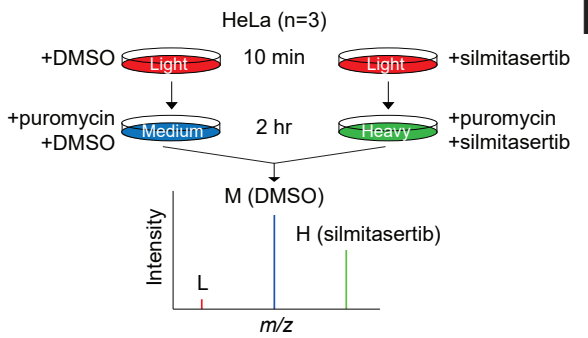

b

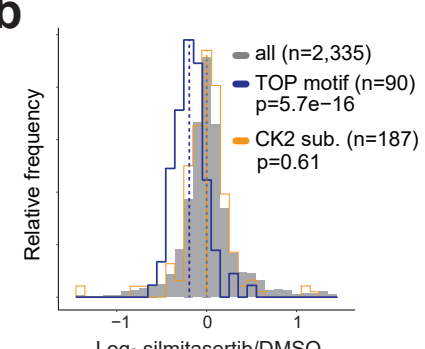

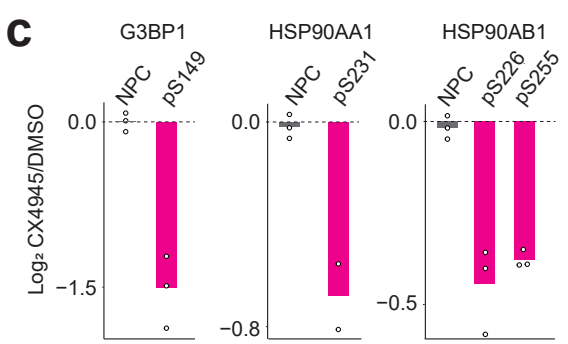

Fig. 2 Quantitative analysis of translational responses and co-translational phosphorylation.

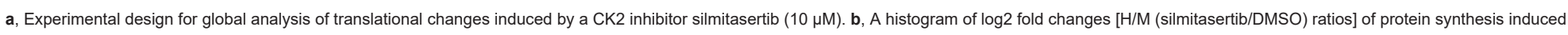

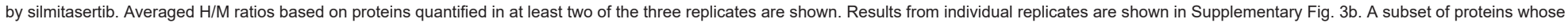

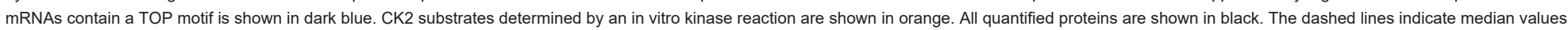

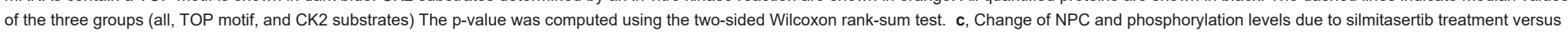

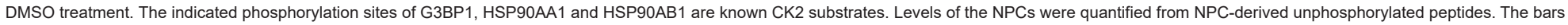
represent averaged values from two or three replicates.

Figure 3

a

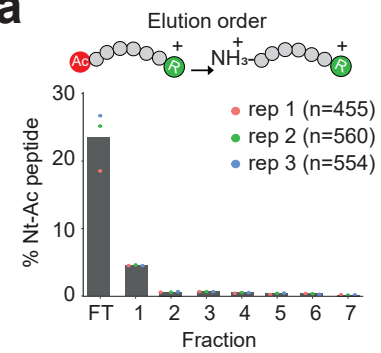

d

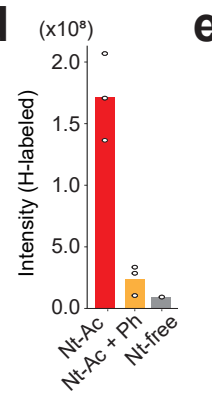

b

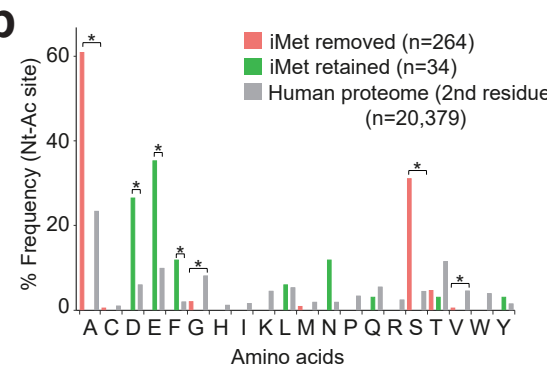

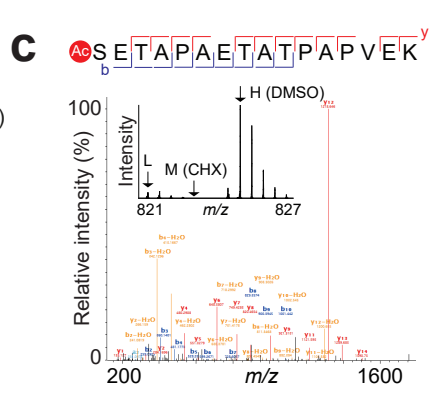

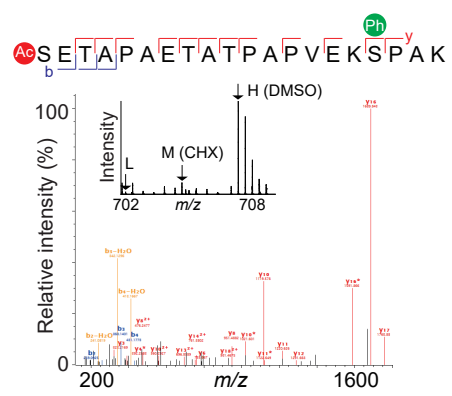

\section{Fig. 3 Characterization of protein Nt-acetylation and phosphorylation on nascent proteins}

a, Enrichment of Nt-acetylated peptides with SCX-based fractionation. The percentage of the number of protein Nt-acetylated peptides in all peptides identified in individual fractions is shown. The bars represent averaged values from three replicates. b. The amino acid frequency at the second residue next to iMet of acetylated protein $\mathrm{N}$-termini based on the absence (pink) or presence (light green) of iMet. Only nascent

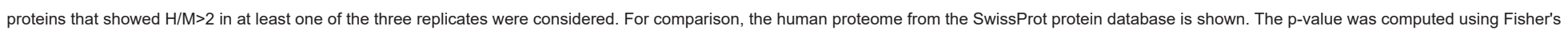
exact test. c, Representative MS/MS spectra of Ac-SETAPAETATPAPVEK (left) and Ac-SETAPAETATPAPVEKpSPAK (right) from histone H1.5. The insets show representative MS spectra of corresponding peptides and demonstrate that the protein Nt-acetylation and adjacent phosphorylation occurred on nascent $\mathrm{H} 1.5$. $\mathbf{d}$, The comparison of $\mathrm{H}$-labeled peptide intensities from Nt-acetylated (red), $\mathrm{Nt}$-acetylated and

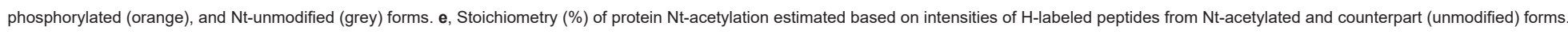
The bars represent averaged values from two or three replicates. $\mathbf{f}$, Experimental design for peptide-pulldown assays using three different peptide probes. Nt-unmodified, Nt-acetylated, or Nt-acetylated and

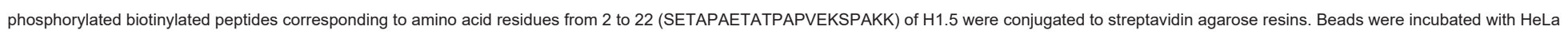

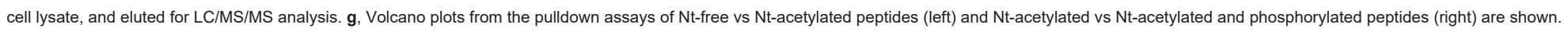




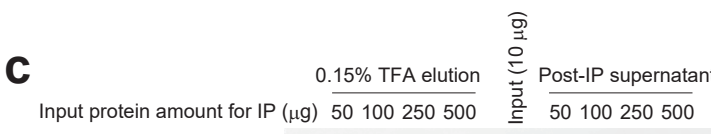
(kDa)
Puromycin $(10 \mu \mathrm{M}) \quad-++$ $\mathrm{CHX}(100 \mu \mathrm{g} / \mathrm{mL})-\mathbf{+}+$ -+
$-\quad+$ (which 5 was not certified $58 y$ peer review) is the author/funder, whohas granted bioRxiv a license to display the preprint in perpetuity. It is made

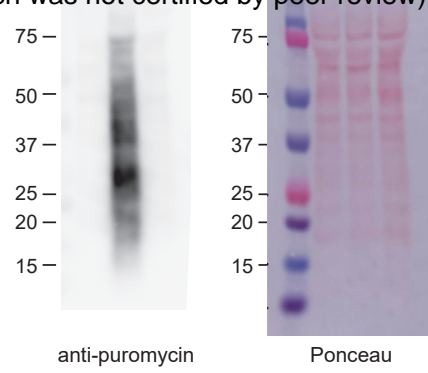
available under aCC-B̄Y-NC 4.0 International license.
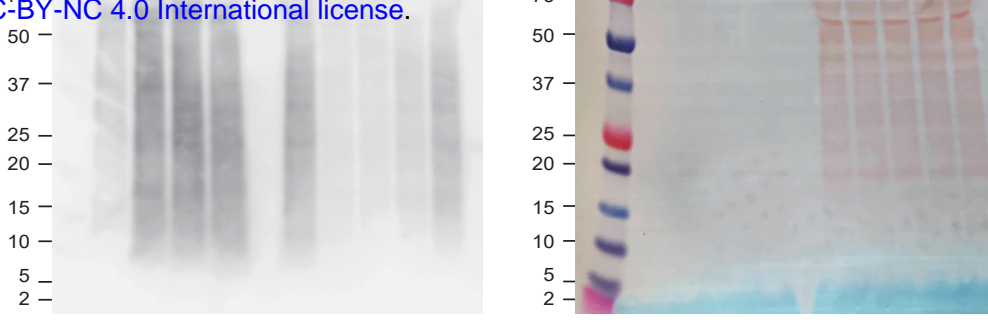

Ponceau

b

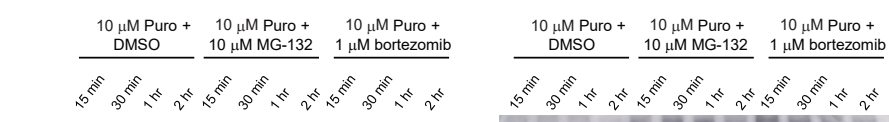

250
150
100
75
50
37
25
20
15
10

二
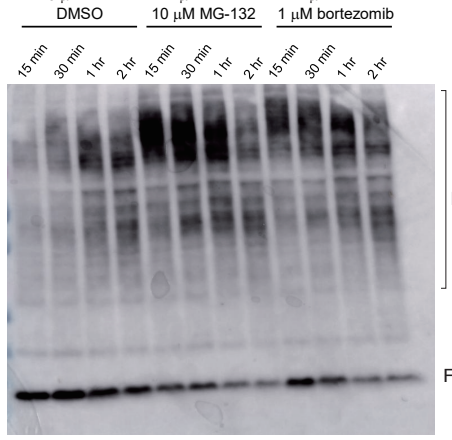

anti-ubiquitin

anti-puromycin

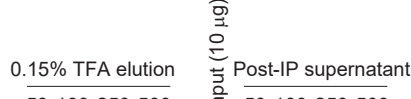

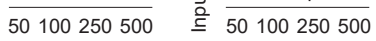
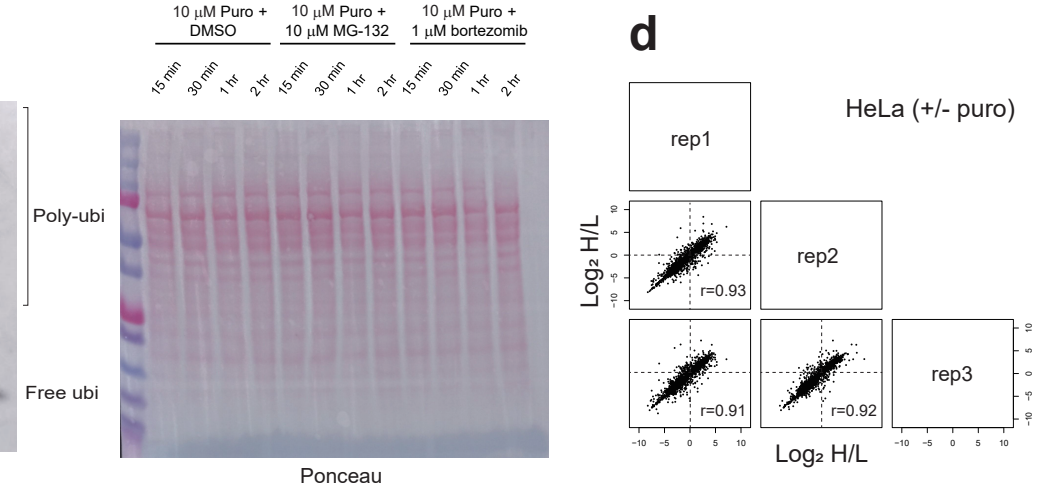

Supplementary Fig. 1 Assessment of puromycin labeling and immunoprecipitation of puromycylated proteins.

a, Puromycin labeling of proteins is translation-dependent. Western blots (left) and Ponceau staining (right) of whole-cell lysates of HeLa cells treated with $10 \mu \mathrm{M}$ puromycin for $2 \mathrm{hr}$, or $10 \mu \mathrm{M}$ puromycin and CHX

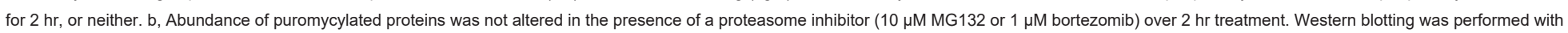
anti-puromycin antibody (left), anti-ubiquitin antibody (middle), and Ponceau staining (right). This result indicates that puromycylated NPCs were not significantly degraded via the ubiquitin-proteasome system in this time frame, while levels of ubiquitinated total cellular proteins were increased in the presence of proteasome inhibitors. c, Puromycylated proteins can be immunoprecipitated. Immunoprecipitation (IP) was

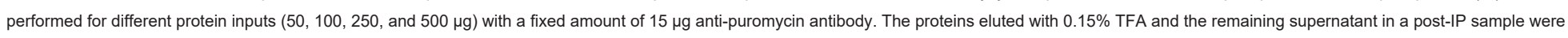

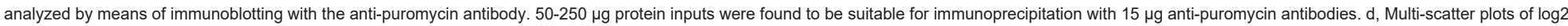
$\mathrm{H} / \mathrm{L}$ ratios from three independent experiments of puromycin $(\mathrm{H})$ and no-puromycin $(\mathrm{L})$ treatments.

\section{Supplementary Fig. 2}

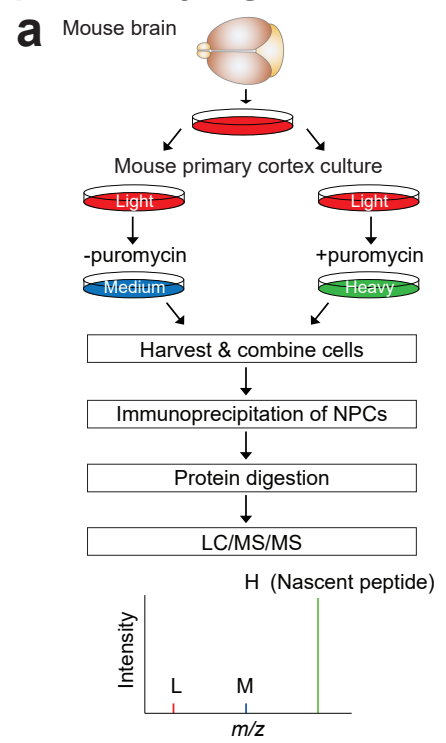

f

$\log _{2}$ DIV5/DIV14 $>0.5, p<0.05$ (122 proteins) b
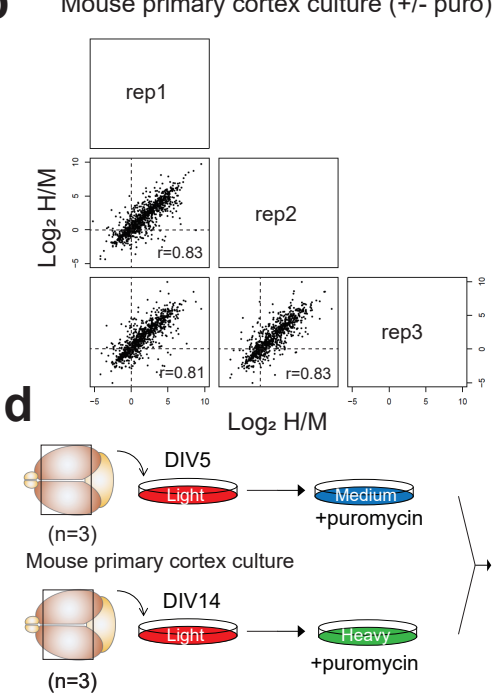
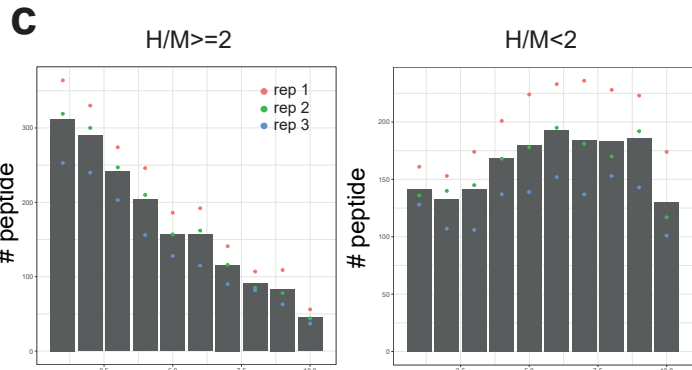

Normalized position within protein e

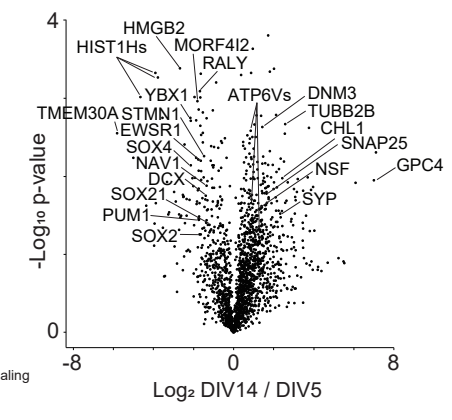

$\log _{2}$ DIV14/DIV5 $>0.5, p<0.05$ (203 proteins)

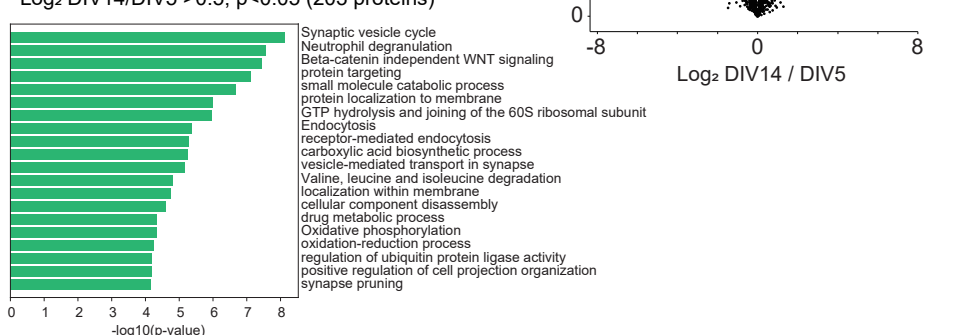

Supplementary Fig. 2 Application of pSNAP to mouse primary cortex culture.

a, Schematic representation of the pSNAP workflow. Mouse primary cortical neurons are pulse labeled for $2 \mathrm{hr}$ with a combination of $10 \mu \mathrm{M}$ puromycin and heavy amino acids or only medium-heavy amino acids.

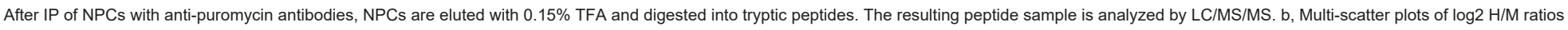

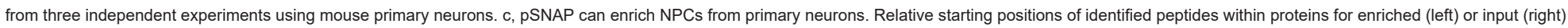

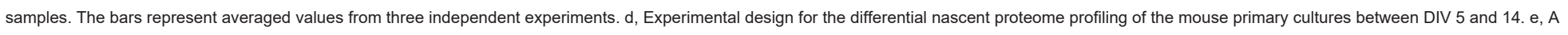

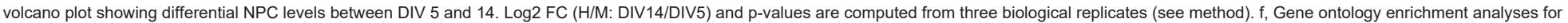




\section{Supplementary Fig. 3}

a

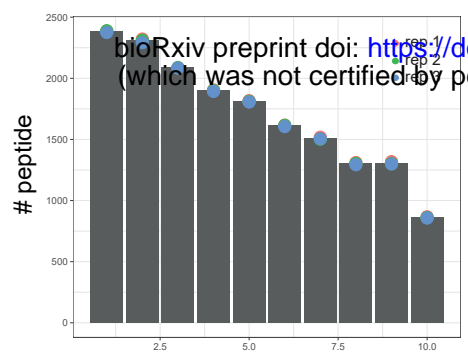

Normalized position within protein b
rep 1
All
rep 2
All
rep 3
All

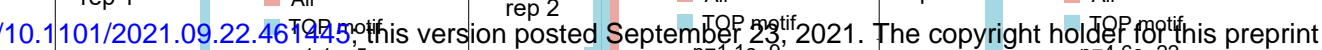
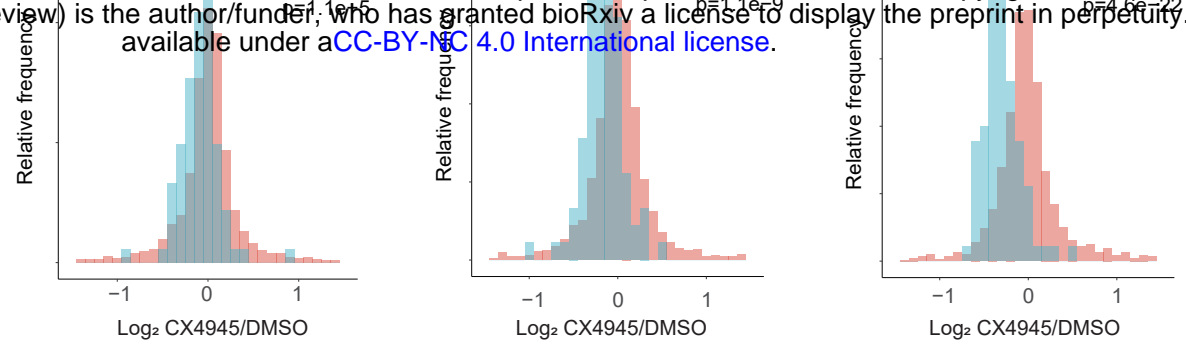

$\log _{2}$ CX4945/DMSO

Supplementary Fig. 3 Quantitative analysis of translational changes associated with CX-4945 treatment.

a, pSNAP can enrich NPCs from HeLa cells treated with DMSO or CX-4945. Relative starting positions of identified peptides (only M- or H-labeled) within proteins for NPC-enriched samples. The bars represent averaged values from three independent experiments. b, Histogram of log2 FCs [H/M (silmitasertib/DMSO) ratios] of protein synthesis induced by silmitasertib. Results from individual replicates are shown. A subset of proteins whose mRNAs contain a TOP motif is shown in light blue. All quantified proteins are shown in pink. The $p$ values were computed using two-sided Wilcoxon rank-sum test.

\section{Supplementary Fig. 4}

a

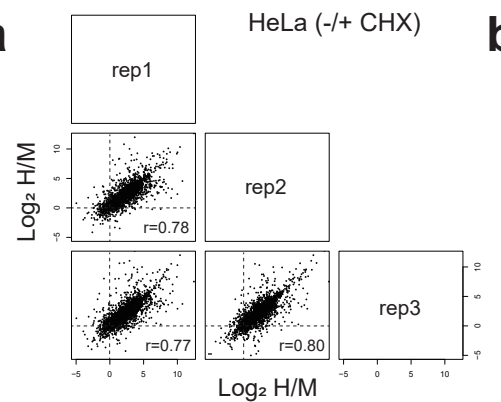

b

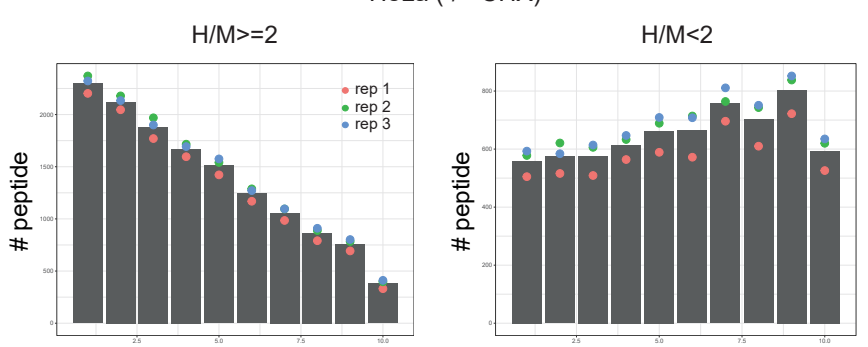

Normalized position within protein

Supplementary Fig. 4 Quantitative analysis of nascent protein N-terminal acetylation.

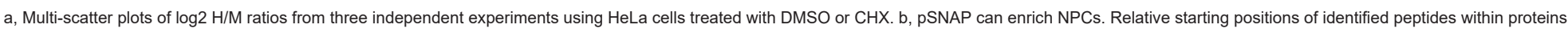

for enriched (left) or input (right) samples. The bars represent averaged values from three independent experiments. 


\section{Methods}

\section{HeLa cell culture and pulse labeling with puromycin and SILAC amino acids}

HeLa cells (ATCC) were cultured in Dulbecco's modified eagle medium (DMEM) (FUJIFILM Wako) supplemented with 10\% fetal bovine serum (FBS) (Thermo Fisher Scientific). Cells were grown to approximately $70-80 \%$ confluence and then used for experiments. For pulse labeling experiments, the cell culture medium was switched to arginine- and lysine-free DMEM (Thermo Fisher Scientific) supplemented with 10\% FBS and $10 \mu \mathrm{M}$ puromycin (FUJIFILM Wako), either "heavy" amino acids $\left[0.398 \mathrm{mM} \mathrm{L}-\left({ }^{13} \mathrm{C}_{6},{ }^{15} \mathrm{~N}_{4}\right)\right.$-arginine (Arg" 10 ") and $0.798 \mathrm{mM} \mathrm{L}-\left({ }^{13} \mathrm{C}_{6},{ }^{15} \mathrm{~N}_{2}\right)$-lysine

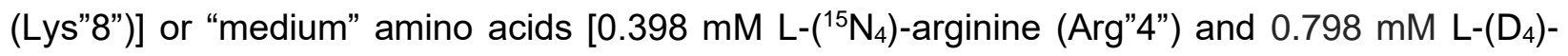
lysine (Lys"4")] (Cambridge Isotope Laboratories), and incubated for $2 \mathrm{hr}$ as previously described elsewhere (Imami et al., 2010, 2018; Uchiyama et al., 2020). For the proof-of-concept experiment (related to Fig. 1b), HeLa cells were pulse-labeled with a combination of $10 \mu \mathrm{M}$ puromycin and 'heavy' amino acids (Arg'10' and Lys'8') for $2 \mathrm{hr}$, while as control, cells were treated with only 'medium-heavy' amino acids (Arg'4' and Lys'4') and puromycin was omitted. To examine the degradation of puromycylated NPCs during puromycin labeling (related to Supplementary Fig. 1b), HeLa cells were treated with $10 \mu \mathrm{M}$ puromycin and either DMSO, $10 \mu \mathrm{M}$ MG-132 (Chemscene) or $1 \mu \mathrm{M}$ bortezomib (FUJIFILM Wako) for $15 \mathrm{~min}, 30 \mathrm{~min}, 60 \mathrm{~min}$ and $120 \mathrm{~min}$. In the silmitasertib experiment (related to Fig. 2a), HeLa cells were pre-incubated with either DMSO or a CK2 inhibitor silmitasertib (MedChemExpress) $(10 \mu \mathrm{M})$ for $10 \mathrm{~min}$, and then pulse-labeled with puromycin for $2 \mathrm{hr}$ in the presence of either DMSO (+'medium-heavy' amino acids) or $10 \mu \mathrm{M}$ silmitasertib (+'heavy' amino acids). For the protein Nt-acetylation profiling (related to Fig. 3a), HeLa cells were pulse-labeled with puromycin for $2 \mathrm{hr}$ in the presence of either $100 \mu \mathrm{g} / \mathrm{mL} \mathrm{CHX}$ (FUJIFILM Wako) (+'medium-heavy' amino acids) or DMSO (+'heavy' amino acids). After pulselabeling, cells were washed twice with ice-cold PBS and collected by centrifugation. Three independent experiments were performed in all proteomic studies. All cells were maintained in an incubator at $37^{\circ} \mathrm{C}$ under humidified $5 \% \mathrm{CO}_{2}$ in air.

\section{Mouse primary cortex cultures and pulse labeling}

31 Primary cultures of cortical neurons were prepared as described with a few modifications (Kaech and Banker, 2006). In brief, cortices were dissected from postnatal day 0 (P0) mice. Cortical neurons were dissociated using neuron dissociation solutions (FUJIFILM Wako) and plated on 35

$34 \mathrm{~mm}$ (DIV7 neuron pulse labeling) or $60 \mathrm{~mm}$ (DIV5 and DIV14 neuron pulse labelling) dishes 
coated with poly-L-lysine (Merck) at a density of $1 \times 10^{5}$ and $2 \times 10^{5} \mathrm{cells} / \mathrm{cm}^{2}$, respectively, in MEM (Thermo Fisher Scientific) supplemented with 10\% horse serum (Thermo Fisher Scientific), 0.6\% D-glucose (Merck), $1 \mathrm{mM}$ sodium pyruvate (Nacalai Tesque) and 1\% penicillin-streptomycin (Thermo Fisher Scientific). Three hours after plating, the media was replaced by growth media consisting of Neurobasal-A medium (Thermo Fisher Scientific) supplemented with B-27 supplement (Thermo Fisher Scientific), GlutaMAX (Thermo Fisher Scientific) and penicillinstreptomycin (Thermo Fisher Scientific). Neurons were maintained at $37^{\circ} \mathrm{C}$ in $5 \% \mathrm{CO}_{2}$ until experiments. For pulse labeling experiments at 7 days in vitro (related to Supplementary Fig. 2) and experiments to assess differential translations between 5 and 14 days in vitro (related to Supplementary Fig. 2d), the cell culture medium was switched to arginine- and lysine-free Neurobasal-A medium (Research Institute for the Functional Peptides) supplemented with B-27 supplement, GlutaMAX, penicillin-streptomycin and either "heavy" amino acids or "medium-heavy" amino acids, and processed as described above. After pulse labeling, cells were washed two times with ice-cold PBS and directly lysed by using lysis buffer [100 mM HEPES-NaOH (pH 7.5), $150 \mathrm{mM} \mathrm{NaCl}, 1 \%$ Nonidet P-40 (NP-40), 1\% protease inhibitor cocktail (Merck)] and further centrifuged at $21,500 \times \mathrm{g}$ for $5 \mathrm{~min}$ at $4^{\circ} \mathrm{C}$ to get rid of the cell debris. The supernatant was further used for immunoprecipitation with anti-puromycin antibody for the detection of the NPCs.

ICR mice were purchased (Shimizu). This study was carried out in accordance with the Guide for the Care and Use of Laboratory Animals from the Society for Neuroscience and was authorized by the Animal Care and Use Committee of Kyoto University.

\section{Immunoprecipitation of NPCs with anti-puromycin antibody}

HeLa cell pellets were lysed with a buffer [100 mM HEPES-NaOH (pH 7.5), $150 \mathrm{mM} \mathrm{NaCl}, 1 \%$ Nonidet P-40 (NP-40), protease inhibitor cocktail (Merck)], and cell debris was removed by centrifugation ( $\left.4^{\circ} \mathrm{C}, 16,000 \times \mathrm{g}, 30 \mathrm{~min}\right)$. The protein concentration was measured using a $\mathrm{BCA}$ assay (Thermo Fisher Scientific), and $250 \mu \mathrm{g}$ (in $250 \mu \mathrm{L}$ ) protein per sample was used for the following IP. For the silmitasertib treatment experiments (related to Fig. 2a), $125 \mu \mathrm{g}$ protein inputs

64 from 'medium-heavy'- and 'heavy'-labeled cells were combined (total $250 \mu \mathrm{g}$ proteins). $62.6 \mu \mathrm{g}$

65 Dynabeads $^{\mathrm{TM}}$ Protein G Magnetic Beads (Thermo Fisher Scientific) and $15 \mu \mathrm{g}$ anti-puromycin 66 antibody (clone 12D10, Merck Millipore) per IP experiment were mixed in PBS-0.02\% Tween 20 67 (PBS-T) and incubated for $30 \mathrm{~min}$ at room temperature with rotation. The supernatant was 68 removed and the beads were washed twice with a conjugation buffer ( $20 \mathrm{mM}$ sodium phosphate 
and $150 \mathrm{mM} \mathrm{NaCl}$ ). To crosslink the beads and the antibodies, the beads were suspended in 5 mM bis(sulfosuccinimidyl)suberate, disodium salt (BS ${ }^{3}$, Wako FUJIFILM) in the conjugation buffer and incubated for $30 \mathrm{~min}$ at $37^{\circ} \mathrm{C}$. To quench the reaction, $50 \mathrm{mM}$ (final concentration) Tris- $\mathrm{HCl}$ $\mathrm{pH} 7.5$ was added and incubation was continued for $15 \mathrm{~min}$ at room temperature. The antibodyconjugated beads were then rinsed with $0.02 \%$ PBS-T, PBS, and the lysis buffer. The antibodyconjugated beads were incubated with $250 \mu \mathrm{g}$ protein input for $1 \mathrm{hr}$ at $4^{\circ} \mathrm{C}$ with slow rotation. The supernatant was transferred to a new tube, and the beads were washed three times with PBS supplemented with $850 \mathrm{mM} \mathrm{NaCl}$. Puromycylated NPCs were eluted from the beads with $100 \mu \mathrm{L}$ $0.15 \%$ trifluoroacetic acid (TFA) (FUJIFILM Wako), and the elution was repeated once more. All TFA eluates were combined and dried in a SpeedVac (Thermo Fisher Scientific).

\section{Protein digestion and SCX fractionation}

The dried samples were resuspended with $100 \mathrm{mM}$ Tris- $\mathrm{HCl}$ pH 9.0 containing $8 \mathrm{M}$ urea. Proteins were reduced with $10 \mathrm{mM}$ dithiothreitol (DTT) (FUJIFILM Wako) for $30 \mathrm{~min}$ at $37^{\circ} \mathrm{C}$, followed by alkylation with $50 \mathrm{mM}$ 2-iodoacetamide (IAA) (FUJIFILM Wako) for $30 \mathrm{~min}$ at room temperature in the dark. The samples were diluted to $2 \mathrm{M}$ urea with $50 \mathrm{mM}$ ammonium bicarbonate. The proteins were digested with $1 \mu \mathrm{g}$ lysyl endopeptidase (LysC) (FUJIFILM Wako) and $1 \mu \mathrm{g}$ trypsin (Promega) overnight at $37^{\circ} \mathrm{C}$ on a shaking incubator. The resulting peptides were acidified with $0.5 \%$ TFA (final concentration), and fractionated with a StageTip containing SDB-XC (upper) and SCX (bottom) Empore disk membranes (GL Sciences) (Adachi et al., 2016). Peptides were eluted from the tip sequentially using 1) $0.5 \%$ TFA and $30 \%$ acetonitrile (ACN) 2) $1 \%$ TFA and $30 \%$ ACN

3) $2 \%$ TFA and $30 \%$ ACN, 4) 3\% TFA and 30\% ACN 5) $3 \%$ TFA, 100 mM ammonium acetate and $30 \%$ ACN 6) $4 \%$ TFA, $500 \mathrm{mM}$ ammonium acetate and $30 \%$ ACN, and 7) $500 \mathrm{mM}$ ammonium acetate and 30\% ACN. For protein Nt-acetylated profiling (related to Fig. 3a), flowthrough and wash [with $0.1 \%$ TFA and $80 \%$ ACN] fractions were combined and measured by LC/MS/MS. The sample solution was evaporated in a SpeedVac and the residue was resuspended in $0.5 \%$ TFA and $4 \% \mathrm{ACN}$.

\section{Peptide pulldown assay}

100 Synthetic peptides were purchased from Synpeptide, and peptide sequences used were as 101 follows: SETAPAETATPAPVEKSPAKK-K(biotin), Ac-SETAPAETATPAPVEKSPAKK-K(biotin), 102 and Ac-SETAPAETATPAPVEKPSPAKK-K(biotin). Synthetic peptides (60 nmol each) were 
incubated with $18 \mu \mathrm{L}$ streptavidin agarose resins (high capacity streptavidin agarose, Thermo Fisher Scientific) per experiment for $2 \mathrm{hr}$ at room temperature in $80 \mu \mathrm{L}$ of lysis buffer (1\% NP-40, $150 \mathrm{mM} \mathrm{NaCl}, 25 \mathrm{mM}$ Tris- $\mathrm{HCl} \mathrm{pH}$ 7.5, and protease and phosphatase inhibitor cocktails). Synthetic peptides bound to agarose resins were incubated with HeLa cell lysate (300 $\mu \mathrm{g}$ in 500 $\mathrm{uL}$ lysis buffer) for $2 \mathrm{hr}$ at $4^{\circ} \mathrm{C}$. Resins were washed three times with $1 \mathrm{~mL}$ of lysis buffer, and proteins bound to resins were eluted with $60 \mu \mathrm{L}$ of elution buffer [12 $\mathrm{mM}$ sodium deoxycholate, $12 \mathrm{mM}$ sodium N-lauroylsarcosinate, $5 \mathrm{mM}$ DTT, $100 \mathrm{mM}$ Tris-HCl pH 9.0]. Afterward, samples were transferred to new tubes and processed for LC/MS/MS analysis as described elsewhere (Masuda et al., 2008)

\section{Mass spectrometry and data-acquisition}

115 Nano-scale reversed-phase liquid chromatography coupled with tandem mass spectrometry (nanoLC/MS/MS) was performed by an Orbitrap Fusion Lumos mass spectrometer (Thermo Fisher Scientific), connected to a Thermo Ultimate 3000 RSLCnano pump and an HTC-PAL autosampler (CTC Analytics, Zwingen, Switzerland) equipped with a self-pulled analytical column (150 mm length $\times 100 \mu \mathrm{m}$ i.d.) (Ishihama et al., 2002) packed with ReproSil-Pur C18-AQ materials (3 $\mu \mathrm{m}$, Dr. Maisch $\mathrm{GmbH}$, Ammerbuch, Germany). The mobile phases consisted of (A) $0.5 \%$ acetic acid and (B) $0.5 \%$ acetic acid and $80 \%$ ACN. Peptides were eluted from the analytical column at a flow rate of $500 \mathrm{~nL} / \mathrm{min}$ with the following gradient: 5-10\% B in $5 \mathrm{~min}, 10-40 \% \mathrm{~B}$ in 60 $\min , 40-99 \%$ B in $5 \mathrm{~min}$, and 99\% for $5 \mathrm{~min}$. The Orbitrap Fusion Lumos instrument was operated in the data-dependent mode with a full scan in the Orbitrap followed by MS/MS scans for 3 sec using higher-energy collisional dissociation (HCD). The applied voltage for ionization was $2.4 \mathrm{kV}$. The full scans were performed with a resolution of 120,000 , a target value of $4 \times 10^{5}$ ions, and a maximum injection time of $50 \mathrm{~ms}$. The MS scan range was $m / z$ 300-1,500. The MS/MS scans were performed with a 15,000 resolution, a $5 \times 10^{4}$ target value, and a 50 ms maximum injection time. The isolation window was set to 1.6, and the normalized HCD collision energy was 30 . Dynamic exclusion was applied for $20 \mathrm{sec}$.

\section{Processing of mass spectrometry data}

134 All raw data files were analyzed and processed by MaxQuant (Cox and Mann, 2008) (v1.6.17.0 135 or v.1.6.2.10), and the database search was performed with Andromeda(Cox et al., 2011) against 136 the SwissProt database (version 2020-10, 42,372 human protein entries) or mouse UniProt 
database (version 2020-3, 55,462 protein entries) spiked with common contaminants and enzyme sequences. Search parameters included two missed cleavage sites and variable modifications such as $\mathrm{L}-\left({ }^{13} \mathrm{C}_{6},{ }^{15} \mathrm{~N}_{4}\right)$-arginine, $\mathrm{L}-\left({ }^{13} \mathrm{C}_{6},{ }^{15} \mathrm{~N}_{2}\right)$-lysine, $\mathrm{L}-\left({ }^{15} \mathrm{~N}_{4}\right)$-arginine, $\mathrm{L}-\left(\mathrm{D}_{4}\right)$-lysine, methionine oxidation, protein $\mathrm{N}$-terminal acetylation and phosphorylation of tyrosine, serine and threonine (only for the silmitasertib experiment related to Fig. 2 and Nt-acetylome experiment related to Fig. 3). Cysteine carbamidomethylation was set as a fixed modification. The peptide mass tolerance was $4.5 \mathrm{ppm}$, and the MS/MS tolerance was $20 \mathrm{ppm}$. The false discovery rate (FDR) was set to $1 \%$ at the peptide spectrum match (PSM) level and protein level. For the SILAC-based protein quantification, a minimum of one ratio count (unique peptide ion) was used for quantification, and the 're-quantify' and 'match between runs' functions were employed. Raw H/M ratios were used for quantification related to Fig. 1, Fig. 3, and Supplementary Fig. 2b while normalized H/M ratios were used for the differential analysis of NPC levels (related to Fig. 2 and Supplementary Fig. 2d). For label-free quantification of peptide pulldown samples (related to Figs. 3f, g), a minimum of two ratio counts was used for quantification. Only proteins quantified in 2 out of the 3 replicates in at least one condition were used for further analysis, and missing values were imputed from a normal distribution of $\log _{2}$ LFQ intensity using a default setting (width 0.3 , down shift 1.8) in Perseus (Tyanova et al., 2016). Volcano plots were generated based on $\log _{2} \mathrm{FC}$ (xaxis) and - $\log _{10} p$-value from two-sided t-test ( $y$-axis). The curve indicates a cut-off for differentially interacting proteins (FDR<0.05, s0: 0.8).

For the differential NPC analysis in mouse primary cortex cultures (related to Supplementary Fig. 2e), normalized $\mathrm{H} / \mathrm{M}$ ratios were $\log _{2}$ transformed and replicates were averaged when they were quantified in at least two of the three replicates. Two-sided one sample t-tests were performed on the experimental data using Perseus (Tyanova et al., 2016) and proteins were considered as 'significantly regulated' when they were below a t-test $p$-value of 0.05 and above 0.5 (a $\log _{2}$ ratio). GO enrichment (Supplementary Fig. 2f) of the significantly regulated proteins were performed using Metascape (Zhou et al., 2019).

\section{Analysis of the positions of identified peptides within protein sequences}

167 To calculate the positions of identified peptides within protein sequences (related to Fig. 1e, 168 Supplementary Fig. 2c, and Supplementary Fig. 4b), we classified peptides into two groups 169 based on their $\mathrm{H} / \mathrm{M}$ ratios; $\mathrm{H} / \mathrm{M} \geqq 2$ (i.e., NPCs) and $\mathrm{H} / \mathrm{M}<2$ (i.e., potential contaminants). The first amino acids within peptides were mapped onto a protein sequence, and their relative positions 
within proteins (from N-term: 0 to C-term: 1) were calculated. For the input samples, all identified peptides were used to compute the positions within proteins. Only $\mathrm{M}$ - and H-labeled peptides were considered to calculate the positions within protein sequences in Supplementary Fig. 3a.

\section{Western blotting}

177 The cell lysates (corresponding to $28 \mu \mathrm{g}$ protein) were re-suspended in LiDS loading sample 178 buffer (Thermo Fisher Scientific) with $50 \mathrm{mM}$ DTT and incubated at $70^{\circ} \mathrm{C}$ for $5 \mathrm{~min}$. The protein 179 samples were loaded onto a 4-12\% gradient SDS-polyacrylamide gel (Thermo Fisher Scientific) 180 and separated using electrophoresis. The proteins were then transferred to a PVDF membrane 181 (Merck Millipore) using a semi-dry western blot transfer system set to a constant current of 200 $182 \mathrm{~mA}$ for $30 \mathrm{~min}$. The membranes were first blocked by incubation in $5 \%(\mathrm{w} / \mathrm{v}) \mathrm{BSA}$ or $5 \%(\mathrm{w} / \mathrm{v})$ 183 skim milk in Tris-buffered saline and $0.1 \%$ tween 20 (TBS-T) and then incubated with antipuromycin antibody (clone 3RH11, Cosmo Bio Co.) diluted 1:5,000, overnight at room temperature. The membrane was washed three times with $0.1 \%$ TBS-T, incubated with HRPconjugated anti-mouse secondary antibody (1: 20,000 dilution) in 0.1\% TBS-T $1 \mathrm{hr}$ at room temperature, washed three times in TBS-T, and developed with ECL reagent (Thermo Fisher Scientific). Ubiquitinated proteins were detected using anti-ubiquitin rabbit antibody (CST) (1: 2,000 dilution) and HRP-conjugated anti-rabbit secondary antibody (1: 10,000 dilution). Chemiluminescence was detected using the luminescent image analyzer ImageQuant LAS-500

\section{References}

Adachi, J., Hashiguchi, K., Nagano, M., Sato, M., Sato, A., Fukamizu, K., Ishihama, Y., and Tomonaga, T. (2016). Improved Proteome and Phosphoproteome Analysis on a Cation Exchanger by a Combined Acid and Salt Gradient. Anal. Chem. 88, 7899-7903.

Cox, J., and Mann, M. (2008). MaxQuant enables high peptide identification rates, individualized p.p.b.-range mass accuracies and proteome-wide protein quantification. Nat. Biotechnol. 26, 200 1367-1372.

201 Cox, J., Neuhauser, N., Michalski, A., Scheltema, R.A., Olsen, J.V., and Mann, M. (2011). 202 Andromeda: a peptide search engine integrated into the MaxQuant environment. J. Proteome 203 Res. 10, 1794-1805.

204 Imami, K., Sugiyama, N., Tomita, M., and Ishihama, Y. (2010). Quantitative proteome and 205 phosphoproteome analyses of cultured cells based on SILAC labeling without requirement of 
serum dialysis. Mol. Biosyst. 6, 594-602.

Imami, K., Milek, M., Bogdanow, B., Yasuda, T., Kastelic, N., Zauber, H., Ishihama, Y., Affects Translation during Mitosis. Mol. Cell 72, 84-98.e9.

210 Ishihama, Y., Rappsilber, J., Andersen, J.S., and Mann, M. (2002). Microcolumns with selfassembled particle frits for proteomics. J. Chromatogr. A 979, 233-239.

212 Kaech, S., and Banker, G. (2006). Culturing hippocampal neurons. Nat. Protoc. 1, 2406-2415.

213 Masuda, T., Tomita, M., and Ishihama, Y. (2008). Phase transfer surfactant-aided trypsin 214 digestion for membrane proteome analysis. J. Proteome Res. 7, 731-740.

215 Moriya, Y., Kawano, S., Okuda, S., Watanabe, Y., Matsumoto, M., Takami, T., Kobayashi, D., Yamanouchi, Y., Araki, N., Yoshizawa, A.C., et al. (2019). The jPOST environment: an integrated proteomics data repository and database. Nucleic Acids Res. 47, D1218-D1224.

218 Okuda, S., Watanabe, Y., Moriya, Y., Kawano, S., Yamamoto, T., Matsumoto, M., Takami, T., 219 Kobayashi, D., Araki, N., Yoshizawa, A.C., et al. (2017). jPOSTrepo: an international standard 220 data repository for proteomes. Nucleic Acids Res. 45, D1107-D1111.

221 Tyanova, S., Temu, T., Sinitcyn, P., Carlson, A., Hein, M.Y., Geiger, T., Mann, M., and Cox, J. 222 (2016). The Perseus computational platform for comprehensive analysis of (prote)omics data. 223 Nat. Methods 13, 731-740.

224 Uchiyama, J., Ishihama, Y., and Imami, K. (2020). Quantitative nascent proteome profiling by 225 dual pulse labeling with O-propargyl-puromycin and stable isotope labeled amino acids. J. 226 Biochem.

227 Zhou, Y., Zhou, B., Pache, L., Chang, M., Khodabakhshi, A.H., Tanaseichuk, O., Benner, C., 228 and Chanda, S.K. (2019). Metascape provides a biologist-oriented resource for the analysis of 229 systems-level datasets. Nat. Commun. 10, 1523. 